\title{
An analysis of radical diffusion in ionic liquids in terms of free volume theory ${ }^{\circledR}$
}

Cite as: J. Chem. Phys. 152, 024502 (2020); https://doi.org/10.1063/1.5138130

Submitted: 13 November 2019 . Accepted: 19 December 2019 . Published Online: 08 January 2020

Dalibor Merunka (iD, and Miroslav Peric (D)

COLLECTIONS

EP This paper was selected as an Editor's Pick

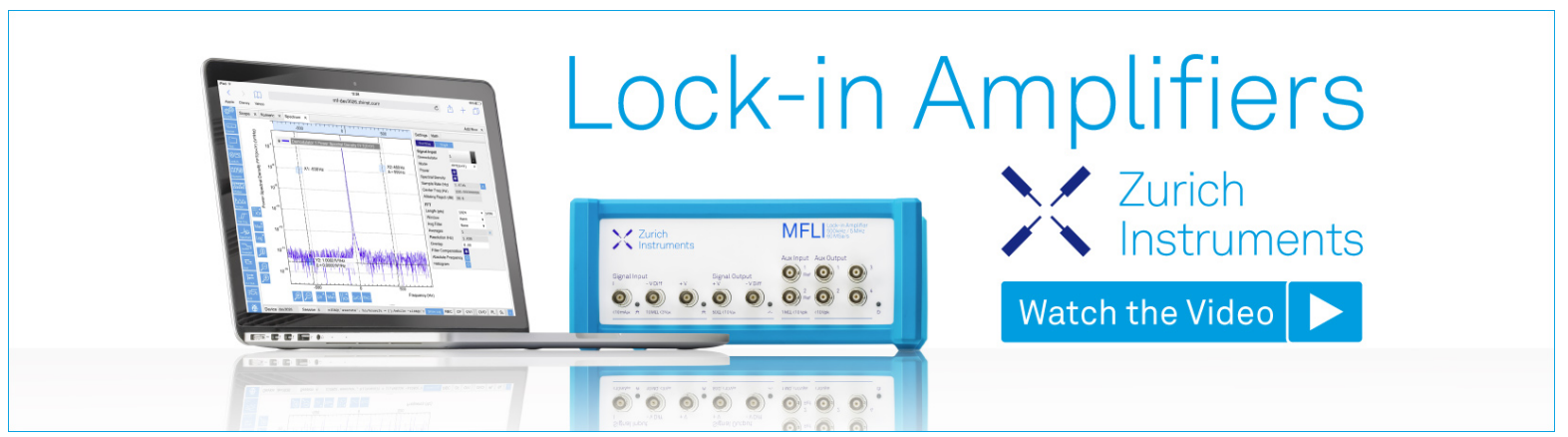




\title{
An analysis of radical diffusion in ionic liquids in terms of free volume theory
}

\author{
Cite as: J. Chem. Phys. 152, 024502 (2020); doi: 10.1063/1.5138130 \\ Submitted: 13 November 2019 - Accepted: 19 December 2019 • \\ Published Online: 8 January 2020
}

\section{Dalibor Merunka' (D) and Miroslav Peric ${ }^{2, a)}$ (iD}

\begin{abstract}
AFFILIATIONS
${ }^{1}$ Division of Physical Chemistry, Ruđer Bošković Institute, Bijenička cesta 54, HR-10000 Zagreb, Croatia

${ }^{2}$ Department of Physics and Astronomy, The Center for Biological Physics, California State University at Northridge, Northridge, California 91330, USA
\end{abstract}

a) Author to whom correspondence should be addressed: miroslav.peric@csun.edu

\begin{abstract}
The Heisenberg spin exchange-dipole-dipole separation method was used to measure the translational diffusion coefficients of the ${ }^{14} \mathrm{~N}$-labeled perdeuterated 2,2,6,6-tetramethyl-4-oxopiperidine-1-oxyl $\left({ }^{14} \mathrm{~N}\right.$-pDTEMPONE) nitroxide spin probe as a function of temperature in two methylimidazolium ionic liquid series, one based on the tetrafluoroborate $\left(\mathrm{BF}_{4}\right)$ anion and another one on the bis(trifluoromethane)sulfonimide (TFSI, Tf2N) anion. The obtained translational diffusion coefficients of ${ }^{14} \mathrm{~N}$-pDTEMPONE were analyzed in terms of the Cohen-Turnbull free volume theory. It was found that the Cohen-Turnbull theory describes, exceptionally well, the translational diffusion of ${ }^{14} \mathrm{~N}$-pDTEMPONE in all the ionic liquids in the measured temperature range. In addition, the Cohen-Turnbull theory was applied to the viscosity and self-diffusion coefficients of the cation and anion-taken from literature-in the same ionic liquids. The critical free volume for the self-diffusion of the cation and anion in a given ionic liquid is the same, which suggests that the diffusion of each ionic pair is coordinated. The critical free volumes for the ${ }^{14} \mathrm{~N}$-pDTEMPONE diffusion, self-diffusion, and viscosity for a given cation were about $20 \%$ greater in the TFSI based ionic liquids than in the $\mathrm{BF}_{4}$ based ionic liquids. It appears that the ratio of the critical free volumes for a given cation between the two series correlates with the ratio of their densities.
\end{abstract}

Published under license by AIP Publishing. https://doi.org/10.1063/1.5138130

\section{INTRODUCTION}

Compared to volatile organic compounds, room temperature ionic liquids (RTILs) have a number of preferred properties, including high electric conductivity, variable polarity, variable hydrophilicity and hydrophobicity, ${ }^{1-3}$ low vapor pressure, high viscosity (from 50 to $1110 \mathrm{cP}$ at room temperature), high thermal stability, and negligible flammability. ${ }^{4}$ Most of these properties foster their industrial use and application. Additionally, the cation and anion components of the ionic liquids may be varied, which means that the ionic liquid properties may be changed according to one's needs and wishes. ${ }^{5,6}$ Therefore, it is not surprising that they are becoming the solvents of choice in many applications, such as organic synthesis, ${ }^{7}$ adsorption, catalysis, ${ }^{8,9}$ electrochemistry, ${ }^{10}$ and analytical chemistry. ${ }^{11}$ For that reason, it is essential to understand the transport properties of RTILs, such as tracer and host (self-) diffusion, so that one can utilize and maximize the benefits of those properties. $^{12}$
Self-diffusion of the cations and anions in RTILs has been explored by pulsed-field gradient nuclear magnetic resonance (PFGNMR) ${ }^{13-17}$ In addition, the tracer diffusion data of a variety of neutral and charged molecules in RTILs measured by PFG- ${ }^{1} \mathrm{H}-\mathrm{NMR}$, together with available literature data, have been used to uncover what characteristics of the solute and solvent are most important in determining tracer diffusion rates. ${ }^{18}$ The diffusion coefficients in all these Refs. 13-18 were analyzed in terms of either the Stokes-Einstein (SE) relationship or the fractional Stokes-Einstein (SE) relationship. ${ }^{16,18}$ In the case of self-diffusion, the significant observation was that regardless of the size difference between the ion components, the diffusion coefficients of the anion and cation of a given RTIL are not much different. In the follow-up arti$\mathrm{cle}^{19}$ to Ref. 18, Maroncelli and Margulis's groups have performed the computational analysis of the tracer diffusion of small neutral and charged solutes in RTILs. They found out that small neutral molecules diffuse much faster than predicted by the SE relationship, while charged ones diffuse much slower due to the existence 
of locally mostly polar-“stiff" — regions and locally mostly apolar"soft"-regions.

When the EPR line broadening method, ${ }^{20}$ which is based on the concentration-induced broadening of the EPR lines, was introduced, it was thought that it would be beneficial for studying the translational diffusion of radicals in viscous biological systems, such as membranes and lipid bilayers. ${ }^{21}$ After thoroughly evaluating the method, Berner and Kivelson ${ }^{22}$ concluded that, after all, the EPR line broadening method was not promising for exploring the fluidity properties of these systems due to an interplay of dipole-dipole (DD) and Heisenberg spin exchange (HSE) interactions whose contributions to the EPR line of a radical in the highly viscous environment could not be separated. In 1997, our group revisited the EPR line broadening method, ${ }^{23}$ and by using nonlinear EPR spectral fitting of the EPR spectrum, we have shown that the spin exchange frequency, which is related to the translational tracer diffusion, can be obtained not just from the broadening of the EPR line but also from the ratio of the amplitudes of the absorption and dispersion line components and the shift of the EPR lines. In this case, the dispersion line component is not the conventional experimental dispersion signal, but it is the dispersion EPR line shape induced by HSE and DD interactions and is opposite for the low- and high-field EPR lines as described in Ref. 24. In a series of articles, ${ }^{25-30}$ our group and Salikhov's group have continued to work on the separation of the HSE and DD interactions so that the concentration induced broadening of the EPR spectrum can be used to measure the translational diffusion of radicals in highly viscous liquids. Finally, we have recently been able to successfully apply the HSE-DD separation EPR method to measure the diffusion coefficients of the ${ }^{14} \mathrm{~N}$ - and ${ }^{15} \mathrm{~N}$ labeled perdeuterated 2,2,6,6-tetramethyl-4-oxopiperidine-1-Oxyl $\left({ }^{14} \mathrm{~N}\right.$-pDTEMPONE and ${ }^{15} \mathrm{~N}$-pDTEMPONE) radicals in 1-ethyl-3methylimidazolium bis(trifluoromethylsulfonyl)imide (Emim TFSI) ionic liquid, glass-forming liquid propylene carbonate, and hydrogen-bonding liquid ethylene glycol. ${ }^{31}$ The translational diffusion of ${ }^{14} \mathrm{~N}$-pDTEMPONE in those liquids was satisfactorily explained by the fractional Stokes-Einstein relation. The diffusion coefficient values of the radicals were also found to approach the values of the self-diffusion coefficients of the liquids at lower temperatures, while at the higher temperatures, the values of the diffusion coefficient of the radicals were smaller than the values of the self-diffusion coefficients in all three liquids.

Apart from the Stokes-Einstein hydrodynamic approach, selfdiffusion and tracer diffusion in liquids can be explained in terms of free volume models. ${ }^{32-36}$ The volume of a fluid is not homogeneous at the molecular level since there is an empty space between the liquid molecules. This empty space, whose geometry is continuously changing due to thermal fluctuations, is commonly called the free volume. One can assume that once a void (hole) larger than some critical size appears in the neighborhood of a molecule, the molecule moves into the hole without any expense of energy. Then, it remains in that hole- "cage"-until its next chance to move on. Although there are several ways to determine the free volume in a liquid, according to Beichel et al., ${ }^{37}$ the most effective experimental method for quantifying the local free volume (holes) is PALS (Positron Annihilation Lifetime Spectroscopy). The free volumes in a number of RTILs have been characterized by PALS. ${ }^{37-40}$ The obtained free volume data are then used to successfully explain the transport properties (self-diffusion, viscosity, and conductivity) of RTILs by the Cohen-Turnbull free volume theory.

In this work, we applied the HSE-DD separation method to measure the translational diffusion coefficients of the ${ }^{14} \mathrm{~N}$ pDTEMPONE nitroxide spin probe as a function of temperature in two methylimidazolium ionic liquid series, one based on the tetrafluoroborate $\left(\mathrm{BF}_{4}\right)$ anion and another one on the bis(trifluoromethane)sulfonimide (TFSI, Tf2N) anion. The obtained diffusion coefficients of ${ }^{14} \mathrm{~N}$-pDTEMPONE were then analyzed in terms of the Cohen-Turnbull free volume theory. Finally, the Cohen-Turnbull free volume analysis of the translational diffusion of the nitroxides was compared to the Cohen-Turnbull free volume theory explanation of the self-diffusion of the cations and anions, and the viscosity of both RTIL series.

\section{EXPERIMENTAL METHODS}

\section{A. Materials}

The spin probe ${ }^{14} \mathrm{~N}$-pDTEMPONE (99 at. \% D) was purchased from $\mathrm{CDN}$ Isotopes and used as received. The ionic liquids 1ethyl-3-methylimidazolium tetrafluoroborate (Emim $\left.\mathrm{BF}_{4}\right)$, 1-butyl3-methylimidazolium tetrafluoroborate $\left(\mathrm{Bmim} \mathrm{BF}_{4}\right)$, 1-hexyl-3methylimidazolium tetrafluoroborate $\left(\mathrm{Hmim} \mathrm{BF}_{4}\right)$, 1-octyl-3methylimidazolium tetrafluoroborate $\left(\mathrm{Omim} \mathrm{BF}_{4}\right)$, 1-decyl-3methylimidazolium tetrafluoroborate (Dmim $\left.\mathrm{BF}_{4}\right)$, 1-ethyl-3methylimidazolium bis(trifluoromethylsulfonyl)imide(Emim TFSI), 1-butyl-3-methylimidazolium bis(trifluoromethylsulfonyl)imide (Bmim TFSI), 1-hexyl-3-methylimidazolium bis(trifluoromethylsulfonyl)imide (Hmim TFSI), 1-octyl-3-methylimidazolium bis (trifluoromethylsulfonyl)imide (Omim TFSI), 1-decyl-3-methylimidazolium bis(trifluoromethylsulfonyl)imide (Dmim TFSI) and 1-dodecyl-3-methylimidazolium bis(trifluoromethylsulfonyl)imide (Ddmim TFSI) were purchased from IOLITEC.

Stock solutions of $36 \mathrm{mM}$ of ${ }^{14} \mathrm{~N}$-pDTEMPONE were prepared by weight in each IL. Then, 12 concentrations of ${ }^{14} \mathrm{~N}$-pDTEMPONE (from $3 \mathrm{mM}$ to $36 \mathrm{mM}$ ) were prepared from the stock solution. Before EPR measurements, the samples were drawn into $5 \mu \mathrm{l}$ capillaries. The bottom end of each capillary was sealed by Heamatocrit sealing compound, while the top end was left open. EPR spectra were measured with a Varian E-109 X-band spectrometer upgraded with a Bruker microwave bridge and a Bruker high-Q cavity. Spectra were acquired using a sweep time of $20 \mathrm{~s}$; sweep width, $50 \mathrm{G}$; time constant, $16 \mathrm{~ms}$; microwave power, $0.5 \mathrm{~mW}$; and modulation amplitude, 0.1 G. A thermocouple connected to an Omega temperature indicator was placed above but very close to the active region of the EPR cavity to avoid reducing the cavity Q-factor. The sample temperature, controlled by a Bruker variable temperature unit, was held stable within $\pm 0.2 \mathrm{~K}$. The temperature increase interval, $5 \mathrm{~K}$, was the same for all samples, while the temperature range was chosen based on the thermal properties of the ionic liquid. The concentrations of ${ }^{14} \mathrm{~N}$-pDTEMPONE were corrected for each temperature using the literature temperature density values for each IL.

\section{B. The HSE-DD separation EPR method}

The HSE-DD separation EPR method has been described in detail previously in Refs. 28 and 31; here, we give a brief overview 
of the method. Due to the use of field modulation and lock-in detection, the experimental EPR spectrum of a nitroxide radical $\mathrm{S}(\mathrm{B})=\mathrm{dR}(\mathrm{B}) / \mathrm{dB}$ is the first derivative of the absorption EPR signal $\mathrm{R}(\mathrm{B})$ with respect to the applied magnetic field B. The EPR spectrum of ${ }^{14} \mathrm{~N}$-pDTEMPONE has three EPR lines because the nitrogen nucleus ${ }^{14} \mathrm{~N}$ has the spin $I=1$. The original spectral function for the absorption EPR spectra of ${ }^{14} \mathrm{~N}$-labeled radicals interacting by HSE and DD interactions can be calculated from the modified Bloch equations, ${ }^{28,29,31}$ and it is given by

$$
R(B)=J_{0} \operatorname{Re}\left[\frac{G(B)}{1-\Lambda G(B)}\right] ; G(B)=\sum_{k=1}^{3} \frac{1}{z_{k}+\Lambda+i\left(B-B_{0}\right)},
$$

where $J_{0}$ is a constant, $\Lambda$ is the coherence transfer rate, $B_{0}$ is the central field line position of the spectrum, and $z_{k}$ is the $k$ th hyperfine line parameter. The line parameters have the forms

$$
z_{1}=\Gamma_{1}-i(A+S / 3), z_{2}=\Gamma_{2}+2 i S / 3, z_{3}=\Gamma_{3}+i(A-S / 3),
$$

where $\Gamma_{k}$ is the spin dephasing rate of the $k$ th line, $A$ is the nitrogen hyperfine splitting, and $S$ is a small relative second-order hyperfine shift.

Experimental EPR spectra are fitted to the first derivative of $R(B)$ from Eqs. (1a) and (1b), using the nonlinear regression command in Mathematica [see Figs. SI 1(a)-SI 1(d) of the supplementary material]. From the fits, we obtain the values of $\Gamma$, $\Lambda$, and $A$ as a function of concentration [see Figs. SI 2(a) and SI 2(b) of the supplementary material and Fig. 2 in Ref. 28]; here, $\Gamma$ is the average linewidth of the hyperfine lines equal to $\left(\Gamma_{1}+\Gamma_{2}+\Gamma_{3}\right) / 3$. Then, for each temperature, from the plots of $\Gamma, \Lambda$, and $A$ vs concentration, we obtain the linear concentration coefficients of the average linewidth $W_{2}$, coherence transfer rate $V_{2}$, and hyperfine splitting $B_{2}$, respectively. The coefficient $B_{2}$ is not considered further since it has a much smaller value than the coefficients $W_{2}$ and $V_{2}$. Using the experimental values of $W_{2}$ and $V_{2}$ together with their numerically calculated theoretical values for different diffusion coefficients [see Figs. SI 2(c) and SI 2(d) of the supplementary material and Fig. 3 in Ref. 28], we find the values of diffusion coefficients $D_{W}$ and $D_{V}$, respectively. Figures SI 2(c) and SI 2(d) of the supplementary material and Fig. 3 in Ref. 28 were obtained by solving the kinematic equations for the spin evolution of a nitroxide pair, considering the nitroxide spin probes as continuously diffusing spherical objects in the hard-core pair potential. Finally, the diffusion coefficient value
$D$ was calculated as the average value $\left(D_{W}+D_{V}\right) / 2$ in the range $D>4 \AA^{2} /$ ns, where $D_{W}$ and $D_{V}$ do not differ much.

\section{RESULTS}

\section{A. ${ }^{14} \mathrm{~N}$-pDTEMPONE translational diffusion in RTILs}

The diffusion coefficient values $D$ of ${ }^{14} \mathrm{~N}$-pDTEMPONE as a function of temperature are shown in Fig. 1(a) $\left(\mathrm{BF}_{4}\right.$ based RTIL series) and Fig. 1(b) (TFSI based RTIL series). The lines are fits to the Vogel-Fulcher-Tammann equation,

$$
D=D_{0} \exp \left(\frac{B}{T-T_{0}}\right)
$$

The fits are excellent, with the correlation coefficients higher than 0.9997. The values of the Vogel-Fulcher-Tammann fit parameters are presented in Table I. For the same cation, the translational diffusion is faster in TFSI RTILs [Fig. 1(b)] than in $\mathrm{BF}_{4}$ RTILs [Fig. 1(a)]. This can be even better observed in Fig. SI 3 of the supplementary material, where all the data are plotted together. In addition, as the chain length of the cation increases, the step decrease in diffusion decreases, becoming almost negligible, especially in the case of TFSI [Fig. 1(b)]. This diffusion saturation implies that the diffusion of ${ }^{14} \mathrm{~N}$-pDTEMPONE in longer chain RTILs is not that much affected by the increasing apolar areas composed of alkyl chains.

According to Cohen and Turnbull, ${ }^{35,36}$ the translational diffusion of a tracer molecule can be described by the Cohen-Turnbull equation,

$$
D=A_{D} \sqrt{T} \exp \left(\frac{-\gamma V^{*}}{V_{f}}\right),
$$

where $\gamma V^{*}$ is the minimum (critical) free volume for the diffusion of a tracer or solvent molecule to occur, $V_{f}$ is the free volume per solvent molecule, and $\gamma$ is a constant of order unity. Usually, it is not easy to obtain a reasonable estimate of $V_{f}$ for a solvent, especially for an ionic liquid. Recently, a series of articles ${ }^{37-40}$ in which the free volumes in several imidazolium ionic liquids were characterized and analyzed by PALS spectroscopy has been published. In Ref. 37, the authors presented an excellent discussion of the relations between different types of volumes that can be defined in solid and liquid
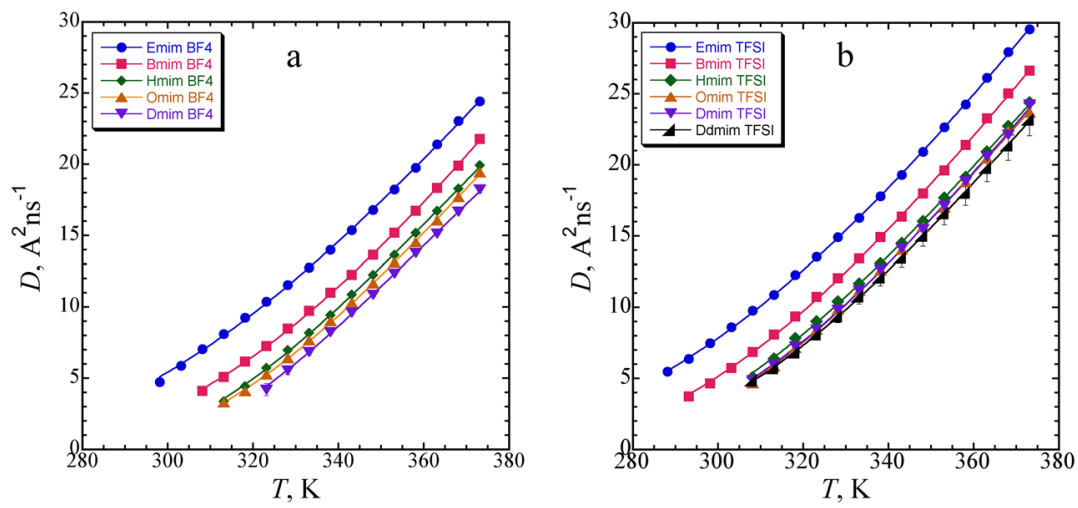

FIG. 1. Diffusion coefficient $D$ of ${ }^{14} \mathrm{~N}$ pDTEMPONE as a function of $T$ in (a) Emim $\mathrm{BF}_{4}$ (blue circle), Bmim $\mathrm{BF}_{4}$ (red

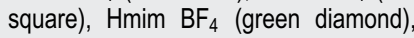
Omim $\mathrm{BF}_{4}$ (brown triangle) and Dmim $\mathrm{BF}_{4}$ (purple inverted triangle), and (b) Emim TFSI (blue circle), Bmim TFSI (red square), Hmim TFSI (green diamond) Omim TFSI (brown triangle), Dmim TFS (purple inverted triangle), and Ddmim (black lower right triangle). The lines are fits to the Vogel-Fulcher-Tammann equation [Eq. (2)]. 
TABLE I. Fit parameters for the diffusion coefficients of ${ }^{14} \mathrm{~N}$-pDTEMPONE in RTILs according to the Vogel-Fulcher-Tammann equation [Eq. (2)].

\begin{tabular}{lcccc}
\hline \hline RTIL & $D_{0}\left(\AA^{2} \mathrm{~ns}^{-1}\right)$ & $B(\mathrm{~K})$ & $T_{0}(\mathrm{~K})$ & $\mathrm{R}$ \\
\hline Emim BF $_{4}$ & $323 \pm 55$ & $513 \pm 56$ & $174 \pm 8$ & 0.9998 \\
$\mathrm{Bmim} \mathrm{BF}_{4}$ & $329 \pm 60$ & $473 \pm 54$ & $199 \pm 8$ & 0.9999 \\
$\mathrm{Hmim} \mathrm{BF}_{4}$ & $154 \pm 24$ & $270 \pm 33$ & $241 \pm 6$ & 0.9998 \\
Omim BF $_{4}$ & $180 \pm 40$ & $303 \pm 49$ & $237 \pm 9$ & 09999 \\
Dmim BF $_{4}$ & $112 \pm 29$ & $209 \pm 47$ & $258 \pm 10$ & 0.9998 \\
Emim TFSI & $461 \pm 60$ & $616 \pm 49$ & $148 \pm 7$ & 0.9999 \\
Bmim TFSI & $301 \pm 49$ & $438 \pm 48$ & $192 \pm 8$ & 0.9999 \\
Hmim TFSI & $236 \pm 56$ & $372 \pm 64$ & $209 \pm 11$ & 0.9998 \\
Omim TFSI & $206 \pm 47$ & $331 \pm 56$ & $219 \pm 10$ & 0.9997 \\
Dmim TFSI & $264 \pm 58$ & $394 \pm 60$ & $208 \pm 10$ & 0.9998 \\
Ddmim TFSI & $254 \pm 39$ & $393 \pm 40$ & $209 \pm 7$ & 0.9999 \\
\hline \hline
\end{tabular}

phases. By using volumes obtained by X-ray diffraction (XRD), the authors created a link between the molecular structure and free volume in RTILs and successfully described the transport properties, such as viscosity and conductivity, of ILs in terms of free volume theory.

The free (hole) volume per molecule $V_{f}$ can be found according to the following equation: ${ }^{37,40}$

$$
V_{f}=\frac{M_{A}}{\rho N_{A}}-V_{m, s c}
$$

where $M_{A}$ is the molar weight, $\rho$ is the density, $N_{A}$ is the Avogadro constant, and $V_{m, s c}$ is the scaled molecular volume. The values of $V_{m, s c}$ for $\mathrm{BF}_{4}^{-}\left(79 \AA^{3}\right)$, TFSI ${ }^{-}\left(233 \AA^{3}\right)$, and $\mathrm{Bmim}^{+}\left(197 \AA^{3}\right)$ are from Ref. 37, while the values of $V_{m, s c}$ for $\mathrm{Emim}^{+}\left(141 \AA^{3}\right), \mathrm{Hmim}^{+}$ $\left(253 \AA^{3}\right), \mathrm{Omim}^{+}\left(309 \AA^{3}\right), \mathrm{Dmim}^{+}\left(365 \AA^{3}\right)$, and $\mathrm{Ddmim}^{+}\left(421 \AA^{3}\right)$ are calculated from $V_{m, s c}$ of $\mathrm{Bmim}^{+}$assuming that the value of $V_{m, s c}$ for $\mathrm{CH}_{2}$ is $28 \AA^{3}{ }^{41}$ It is assumed that the total ionic molecule volume is the sum of the ionic volumes of the constituent ions. For the values of $V_{m, s c}$ and $M_{A}$ for each RTIL, see Table SI 1. The values of $\rho$ are taken from the literature, and the equations used for the calculation of $\rho$ for each RTIL and source references are given in the supplementary material section.
TABLE II. Fit parameters for the diffusion coefficients of ${ }^{14} \mathrm{~N}-\mathrm{pDTEMPONE}$ in RTILs according to the Cohen-Turnbull equation [Eq. (3)].

\begin{tabular}{lccc}
\hline \hline RTIL & $A_{D}\left(\AA^{2} \mathrm{~ns}^{-1}\right)$ & $\gamma \mathrm{V}^{*}\left(\AA^{3}\right)$ & $\mathrm{R}$ \\
\hline Emim BF $_{4}$ & $92.5 \pm 3.1$ & $204.2 \pm 1.5$ & 0.998 \\
$\mathrm{Bmim} \mathrm{BF}_{4}$ & $83.2 \pm 2.9$ & $217.3 \pm 1.3$ & 0.998 \\
$\mathrm{Hmim} \mathrm{BF}_{4}$ & $63.6 \pm 3.0$ & $220.1 \pm 2.4$ & 0.996 \\
Omim BF $_{4}$ & $40.3 \pm 2.2$ & $210.0 \pm 2.8$ & 0.997 \\
Dmim BF $_{4}$ & $25.1 \pm 1.6$ & $196.4 \pm 3.5$ & 0.997 \\
Emim TFSI & $33.1 \pm 0.7$ & $231.0 \pm 1.4$ & 0.999 \\
Bmim TFSI & $37.9 \pm 1.3$ & $262.0 \pm 2.4$ & 0.998 \\
Hmim TFSI & $30.9 \pm 1.4$ & $265.0 \pm 3.4$ & 0.998 \\
Omim TFSI & $26.0 \pm 1.2$ & $261.4 \pm 3.6$ & 0.998 \\
Dmim TFSI & $20.7 \pm 0.7$ & $249.8 \pm 2.9$ & 0.999 \\
Ddmim TFSI & $16.6 \pm 0.4$ & $242.1 \pm 2.0$ & 0.999 \\
\hline \hline
\end{tabular}

The diffusion coefficient values $D$ divided by the square root of the temperature of ${ }^{14} \mathrm{~N}$-pDTEMPONE as a function of the free volume are shown in Fig. 2(a) $\left(\mathrm{BF}_{4}\right.$ based RTIL series) and Fig. 2(b) (TFSI based RTIL series). For a given cation, the available free volume is about $50 \%$ larger in the TFSI RTILs than that in the $\mathrm{BF}_{4}$ RTILs [Fig. SI 4]. The lines in the figures are fits to the Cohen-Turnbull equation. The fit parameters for the diffusion coefficients of ${ }^{14} \mathrm{~N}-\mathrm{pDTEMPONE}$ in RTILs and the correlation coefficients, which are greater than 0.996 , are given in Table II. Figure 3(a) shows the values of $\gamma V^{*}$ for the diffusion of ${ }^{14} \mathrm{~N}$-pDTEMPONE in both RTIL series (closed and open blue circles) as a function of the number of carbons in the cation alkyl chain. The values of $\gamma V^{*}$ in the TFSI RTIL series are greater than those in the $\mathrm{BF}_{4}$ RTIL series by about $20 \%$, as shown in Fig. 3(b). This observation is in accordance with the conclusion of Beichel et $a l .{ }^{37}$ that the critical free volume increases with the molecular size.

\section{B. Cation and anion translational self-diffusion in RTILs}

The self-diffusion coefficients of cations $D_{+}$and anions $D_{-}$ in $\mathrm{BF}_{4}{ }^{15,17}$ and TFSI $^{13,14,16}$ RTILs measured by a pulsed-gradient
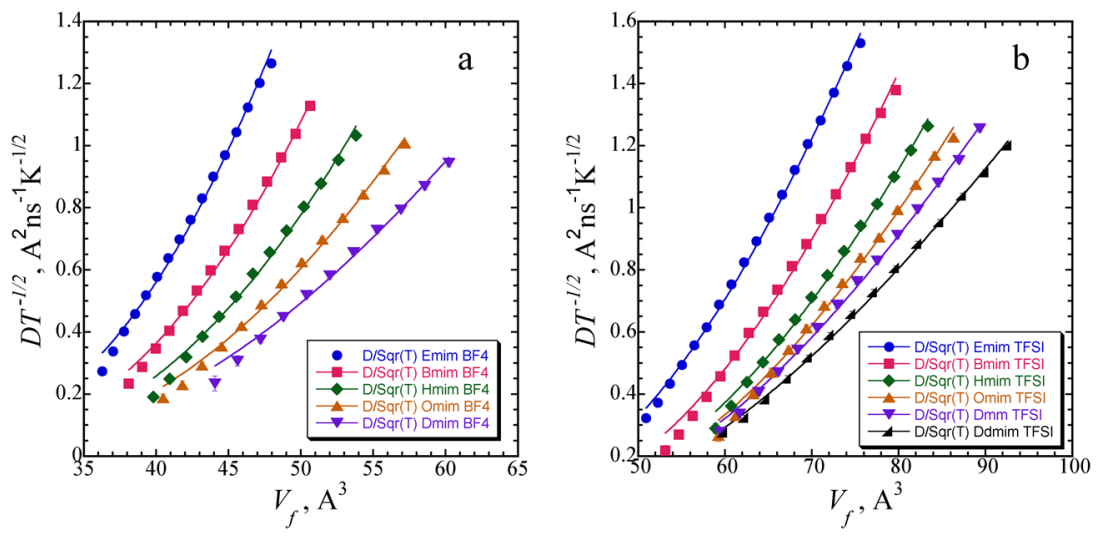

FIG. 2. Diffusion coefficient $D$ over the square root of temperature of ${ }^{14} \mathrm{~N}$ pDTEMPONE as a function of $V_{f}$ in (a) Emim $\mathrm{BF}_{4}$ (blue circle), Bmim $\mathrm{BF}_{4}$ (red square), $\mathrm{Hmim} \mathrm{BF}_{4}$ (green diamond), Omim $\mathrm{BF}_{4}$ (brown triangle), and Dmim $\mathrm{BF}_{4}$ (purple inverted triangle), and (b) Emim TFSI (blue circle), Bmim TFSI (red square), Hmim TFSI (green diamond) Omim TFSI (brown triangle), Dmim TFSI (purple inverted triangle), and Ddmim (black lower right triangle). The lines are fits to the Cohen-Turnbull equation [Eq. (3)]. 

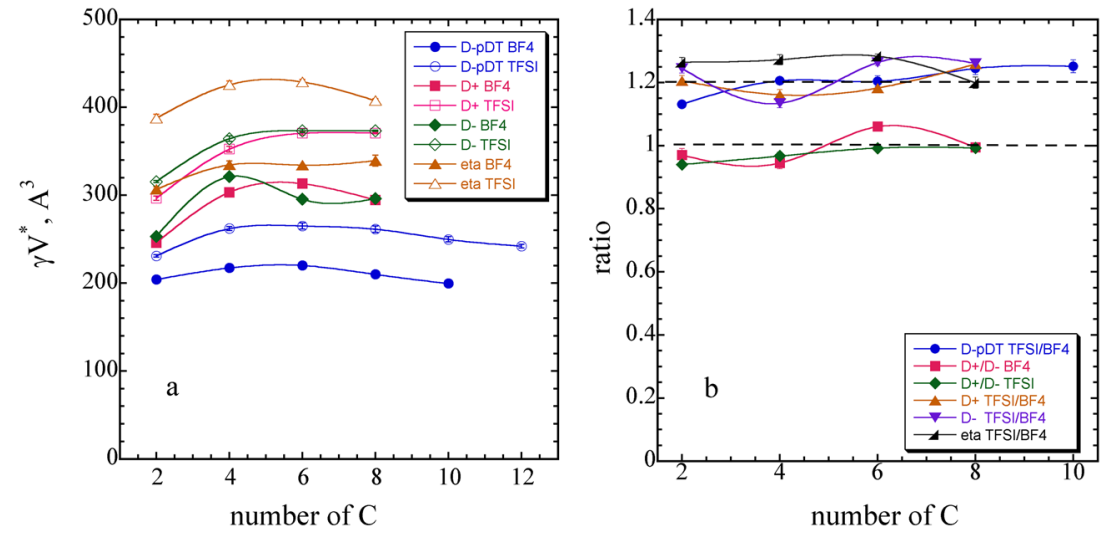

FIG. 3. (a) Critical free volume $\gamma V^{*}$ as a function of the number of carbons in the cation alkyl chain for $D$ of ${ }^{14} \mathrm{~N}-\mathrm{pDTEMPONE}$ in the $\mathrm{BF} \mathrm{F}_{4}$ series (blue filled circle), $\mathrm{D}$ of ${ }^{14} \mathrm{~N}$-pDTEMPONE in the TFSI series (blue unfilled circle), $D_{+}$in the $\mathrm{BF}_{4}$ series(red filled square), $D_{+}$in the TFSI series (red unfilled square), $D_{-}$in the $B F_{4}$ series (green filled diamond), $\mathrm{D}_{-}$in the TFSI series (green unfilled diamond), $\eta$ in the $\mathrm{BF}_{4}$ series (brown filled triangle), and $\eta$ in the TFSI series (brown unfilled triangle). (b) The ratio of $\gamma V^{*}$ from D of ${ }^{14} \mathrm{~N}$-pDTEMPONE in TFSI and $\mathrm{BF}_{4}$ RTILs (blue filled circle), $\mathrm{D}_{+} / \mathrm{D}_{-}$in $\mathrm{BF}_{4} \mathrm{RTILs}$ (red filled square), $\mathrm{D}_{+} / \mathrm{D}_{-}$in TFSI RTILs (green filled diamond), $\mathrm{D}_{+}$in TFSI and

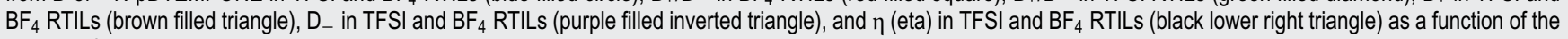
number of carbons in the cation. The lines are to guide the eye.

spin-echo NMR method are taken from the literature. Figure 4(a) shows the self-diffusion coefficients of cations $D_{+}$and anions $D_{-}$ divided by the square root of temperature for the $\mathrm{BF}_{4}$ based RTIL series $\left(C_{2}\right.$ to $\left.C_{8}\right)$ as a function of free volume, while Fig. $4(\mathrm{~b})$ shows the same thing for the TFSI series. The lines are fits to the Cohen-Turnbull equation (3). The obtained parameters for the fits are also given in Table III. From the correlation coefficients in Table III and Figs. 4(a) and 4(b), it can be observed that the fits are excellent. The largest difference in the coefficients of diffusions of the cations and anions occurs in Emim TFSI, then, in decreasing order, in Bmim TFSI, Emim $\mathrm{BF}_{4}$, Omim $\mathrm{BF}_{4}$, and Hmim TFSI, while in the remaining liquids, the difference is negligible.

The minimum critical free volumes for the diffusion of cations and anions $\gamma V^{*}$ have been presented in Fig. 3(a) and Table III. Similarly, as in the case of the values of $\gamma V^{*}$ for ${ }^{14} \mathrm{~N}$-pDTEMPONE
[Fig. 3(a) and Table II], the values of $\gamma V^{*}$ for the self-diffusion of the cations and anions for the same cation are again greater in the TFSI RTIL series than those in the $\mathrm{BF}_{4}$ RTIL series by about $20 \%$ as shown in Fig. 3(b). Interestingly, the critical free volumes for the diffusion of the cations and anions are about the same in all RTILs [Fig. 3(b)]. The ratio of the critical free volumes for the diffusion of cations and anions is close to unity, within five percent.

\section{Viscosity of $\mathrm{BF}_{4}$ and TFSI RTILs in terms of free volume}

It has already been shown that empirical two-parameter Cohen-Turnbull equations are very good descriptions of the viscosity of certain ILs. ${ }^{37-39}$ Since according to the Stokes-Einstein equation $\eta \propto \frac{k_{B} T}{D}$, the Cohen-Turnbull equation for viscosity [Eq. (3)] becomes
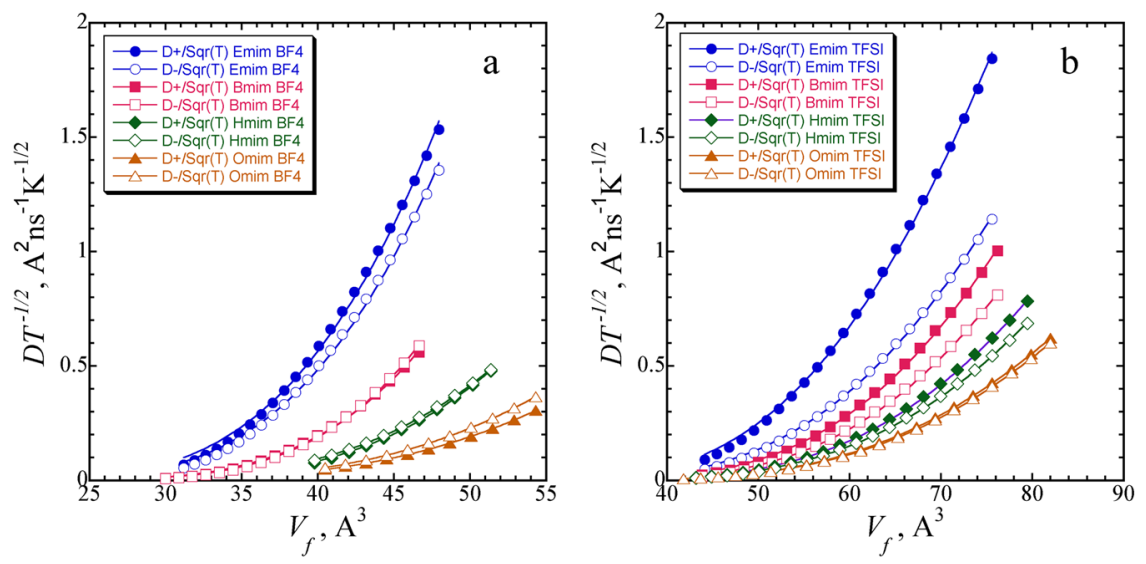

FIG. 4. (a) Self-diffusion coefficients of cations $D_{+}$(closed symbols) and anions $D_{-}$(open symbols) over the square root of temperature as a function of $V_{f}$ in Emim $\mathrm{BF}_{4}$ (circles), Bmim $\mathrm{BF}_{4}$ (squares), $\mathrm{Hmim} \mathrm{BF}_{4}$ (diamonds), and Omim $\mathrm{BF}_{4}$ (triangles); (b) self-diffusion coefficients of cations $D_{+}$(closed symbols) and anions $D_{-}$(open symbols) as a function of $V_{f}$ in Emim TFSI (circles), Omim TFSI (squares), Hmim TFSI (diamonds), and Dmim TFSI(triangles). The lines are fits to the Cohen-Turnbull equation [Eq. (3)]. 
TABLE III. Fit parameters for the diffusion coefficients of cations, $D_{+}$, and anions, $D_{-}$, in RTILs according to the CohenTurnbull equation [Eq. (3)].

\begin{tabular}{lrrrrrr}
\hline \hline RTIL & $A_{D+}\left(\AA^{2} \mathrm{~ns}^{-1}\right)$ & $\gamma \mathrm{V}^{*}\left(\AA^{3}\right)$ & $\mathrm{R}$ & $A_{D-}\left(\AA^{2} \mathrm{~ns}^{-1}\right)$ & $\gamma \mathrm{V}^{*}\left(\AA^{3}\right)$ & $\mathrm{R}$ \\
\hline Emim BF $_{4}$ & $264 \pm 20$ & $245.9 \pm 3.4$ & 0.999 & $272 \pm 21$ & $253.3 \pm 3.5$ & 0.999 \\
$\mathrm{Bmim} \mathrm{BF}_{4}$ & $371 \pm 35$ & $302.4 \pm 4.2$ & 0.999 & $579 \pm 47$ & $321.1 \pm 3.6$ & 0.999 \\
Hmim BF $_{4}$ & $214 \pm 14$ & $313.2 \pm 3.2$ & 0.999 & $153.1 \pm 7.2$ & $295.4 \pm 2.2$ & 0.999 \\
Omim BF $_{4}$ & $68.9 \pm 0.9$ & $294.4 \pm 0.6$ & 0.999 & $85.4 \pm 1.6$ & $296.1 \pm 0.9$ & 0.999 \\
Emim TFSI & $94.6 \pm 3.6$ & $296.6 \pm 2.6$ & 0.999 & $74.9 \pm 2.4$ & $315.4 \pm 2.2$ & 0.999 \\
Bmim TFSI & $103.5 \pm 4.8$ & $352.3 \pm 3.2$ & 0.999 & $97.6 \pm 4.1$ & $364.3 \pm 3.0$ & 0.999 \\
Hmim TFSI & $83.3 \pm 2.1$ & $370.4 \pm 1.8$ & 0.999 & $75.8 \pm 2.1$ & $373.3 \pm 2.0$ & 0.999 \\
Omim TFSI & $57.2 \pm 0.5$ & $370.6 \pm 0.7$ & 0.999 & $57.1 \pm 0.5$ & $373.3 \pm 0.6$ & 0.999 \\
\hline \hline
\end{tabular}
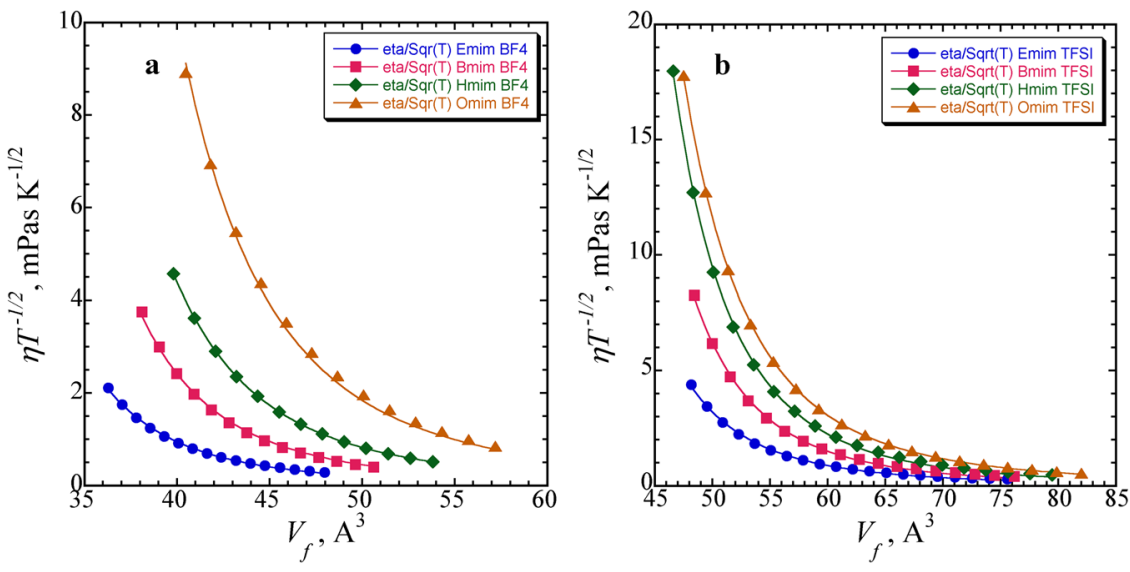

FIG. 5. Viscosity over the square root of temperature of (a) Emim $\mathrm{BF}_{4}$ (blue circle), $\mathrm{Bmim} \mathrm{BF}_{4}$ (red square), $\mathrm{Hmim} \mathrm{BF}_{4}$ (green diamond), and $\mathrm{Omim} \mathrm{BF}_{4}$ (brown triangle), and (b) Emim TFSI (blue circle), Bmim TFSI (red square), Hmim TFSI (green diamond), and Omim TFSI (brown triangle) as a function of $V_{f}$. The lines are fits to the Cohen-Turnbull equation [Eq. (5)].

$$
\eta=A_{\eta} \sqrt{T} \exp \left[\frac{\gamma V^{*}}{V_{f}}\right] .
$$

Here, the viscosity values of both RTIL series taken from the literature are plotted as a function of free volume [Figs. 5(a) and 5(b)]. The viscosities of the $\mathrm{BF}_{4}$ RTILs were calculated according to Table II from Ref. 24, while the viscosities of the TFSI RTILs were calculated by using Table SI 3. The lines in Figs. 5(a) and 5(b) are the fits to the Cohen-Turnbull equation [Eq. (5)]. The fitting parameters are presented in Table IV. In addition, the critical volumes $\gamma V^{*}$ (third

TABLE IV. Fit parameters for the viscosity of RTILs according to the Cohen-Turnbull equation [Eq. (5)].

\begin{tabular}{lccc}
\hline \hline RTIL & $A_{\eta} 10^{5}(\mathrm{mPas})$ & $\gamma \mathrm{V}^{*}\left(\AA^{3}\right)$ & $\mathrm{R}$ \\
\hline Emim BF $_{4}$ & $44.5 \pm 3.4$ & $306.6 \pm 2.9$ & 0.999 \\
$\mathrm{Bmim} \mathrm{BF}_{4}$ & $56.4 \pm 6.3$ & $334.5 \pm 4.5$ & 0.999 \\
$\mathrm{Hmim} \mathrm{BF}_{4}$ & $103.6 \pm 0.6$ & $334.1 \pm 0.3$ & 0.999 \\
Omim BF $_{4}$ & $208 \pm 30$ & $339.3 \pm 6.1$ & 0.999 \\
Emim TFSI & $139 \pm 11$ & $387.7 \pm 4.1$ & 0.999 \\
Bmim TFSI & $125 \pm 10$ & $425.7 \pm 4.1$ & 0.999 \\
Hmim TFSI & $174.2 \pm 9.9$ & $428.9 \pm 2.7$ & 0.999 \\
Omim TFSI & $385.4 \pm 8.1$ & $407.0 \pm 2.3$ & 0.999 \\
\hline \hline
\end{tabular}

column in Table IV) are shown in Fig. 3(a), while the ratio of the critical volumes in TFSI and $\mathrm{BF}_{4}$ RTILs for a given cation is plotted in Fig. 3(b). Again, for a given cation, the critical free volume in the TFSI series is greater by about $20 \%$ than the one in the $\mathrm{BF}_{4}$ series.

\section{DISCUSSION AND CONCLUSIONS}

The translational diffusion of ${ }^{14} \mathrm{~N}$-pDTEMPONE in the studied RTILs can be quite well described in terms of $V_{f}$ within the context of the Cohen-Turnbull theory [Eq. (3)]. The values of $\gamma V^{*}$ in the $\mathrm{BF}_{4}$ series [Fig. 3(a) and Table II] are very close to each other. The spread is from 199.6 to $220.1 \AA^{3}$. The values of $\gamma V^{*}$ in the TFSI series are just slightly greater with a spread from 231.0 to $265.0 \AA^{3}$ [Fig. 3(a) and Table II]. As expected, the critical free volume is the smallest in Emim RTILs. The curves for both series in Fig. 3(a) have weak maxima in Bmim and Hmim RTILs, but a likely conclusion is that the values of $\gamma V^{*}$ do not change that much as a function of the number of $\mathrm{C}$ in the cation alkyl chain. In other words, there is no increase in $\gamma V^{*}$ with the increasing number of C. These results mean that the tracer molecule ${ }^{14} \mathrm{~N}$-pDTEMPONE, which is neutral, needs a similar critical free volume in each given series, which appears to be a reasonable physical picture for tracer translation. 
For more than a decade, it has been known that RTILs form nanostructures. $^{42-44}$ In the case of RTILs with the alkyl chains shorter than $\mathrm{C}_{4}$, due to their electrostatic interactions, anions and cations are homogeneously distributed, and alkyl chain aggregation is minimal. ${ }^{44}$ Starting with the butyl chain length $\left(\mathrm{C}_{4}\right)$, the alkyl chain length enables the chains to form bicontinuous spongelike nanostructures $^{44}$ (see Fig. 11 in Ref. 44). The size of nanostructured soft apolar regions increases with the length of the cation alkyl chain. ${ }^{2,42,43}$ PALS gives only the hole radius distribution in the bulk ionic liquid; ${ }^{38}$ as far as we know, there are no such distributions for each domain. Therefore, if one assumes both homogeneous hole radius distribution and diffusion throughout the bulk ionic liquid, the growth of bicontinuous apolar nanodomains may not change much the overall diffusion of the spin probe. Consequently, the saturation of the diffusion of ${ }^{14} \mathrm{~N}$-pDTEMPONE in longer chain RTILs [Figs. 1(a) and 1(b)] and the slight cation alkyl chain dependence of $\gamma V^{*}$ for RTILs with $\mathrm{C}_{4}$ and above (Fig. 3) are then expected due to the growth of the apolar soft domains and the faster diffusion through them. The presented view of the diffusion of ${ }^{14} \mathrm{~N}$-pDTEMPONE is in agreement with the findings on the diffusion of small neutral molecules in RTILs. ${ }^{19}$ Using MD simulations, Araque et al. ${ }^{19}$ found out that small neutral molecules sample both polar and apolar RTIL domains. According to Araque et al., the neutral probe is caged in the polar stiff regions, and thereby it diffuses slowly, while in the less polar soft regions, the neutral probe experiences fast diffusive jumps. In other words, the translational diffusion of ${ }^{14} \mathrm{~N}$-pDTEMPONE speeds up in apolar nanodomains so that the overall diffusion does not change noticeably.

The self-diffusion of the cations and anions of both series can also be described as a function of $V_{f}$ using the Cohen-Turnbull equation. Again, the fitted values of the critical free volume are noticeably lower in the case of Emim RTILs [Fig. 3(a) and Table III] compared to the remaining RTILs. After four carbons in the alkyl chain, the values of the critical free volume appear almost constant. It has been observed that the self-diffusion coefficients of the cation and anion of a given RTIL are, in many cases, similar, even in the case of substantial size differences. ${ }^{18}$ Here, we observe the similarity in the case of three (Bmim $\mathrm{BF}_{4}, \mathrm{Hmim} \mathrm{BF}_{4}$, and Omim TFSI) out of eight RTILs [Figs. 4(a) and 4(b)]. From the ratio of $\gamma V^{*}$ for $D_{+}$ and $D_{-}$in a given RTIL, which is close to unity (ranges from 0.94 to 1.06), it can be concluded that the values of $\gamma V^{*}$ for the cation and anion of a given RTIL appear to be almost the same [Table III and Fig. 3(b)] even in the case of Emim TFSI, in which the self-diffusion of $D_{-}$is about $70 \%$ faster than the self-diffusion of $D_{+}$[Fig. 4(b)]. In addition, if the self-diffusion coefficients $D_{+}$and $D_{-}$are added and then fitted to the Cohen-Turnbull equation [Eq. (3), Figure SI 5], the values of $\gamma V^{*}$ are the average of the values of $\gamma V^{*}$ for the cations and anions (Table SI 4).

The Cohen-Turnbull equation describes well the viscosity behavior of the studied RTILs in terms of free volume [Figs. 5(a) and 5(b) and Table IV]. Although the values of the critical free volume for viscosity are larger compared to the other two transport properties for both series [Fig. 3(a)], the overall behavior is the same as in the other two cases. The values of $\gamma V^{*}$ do not change noticeably with the length of the cation alkyl chain, and the ratio of the critical volumes in TFSI and $\mathrm{BF}_{4}$ RTILs is still close to 1.2 [Fig. 3(b)]. The ratio seems not to be related to the ratio of the free volumes between the two series (Fig. SI 4) since the free volumes in the TSFI based RTILs are about $50 \%$ greater than those in the $\mathrm{BF}_{4}$ series. It appears that the ratio of the critical free volumes in the two series is related to the ratio of the corresponding densities (Fig. SI 4), which is also close to 1.2. There is no such relation among the members of each series as the critical volumes have almost the same values for the members of each RTIL series (Fig. 3), while their densities are quite different (see Table SI 4 of the supplementary material). At the moment, the relation of the ratio of the critical volumes and the corresponding densities for the studied RTIL series is empirical. It would be interesting to find out whether there is a similar relation between other RTIL series. Since the only difference between the two series is the anion, there is no doubt that the nature of the anion is responsible for this relation. Possibly, the anion size affects the hole radius distribution, which then affects the translation diffusion. Since the size of the TFSI anion is greater than the size of the $\mathrm{BF}_{4}$ anion, the higher critical volume values for the TFSI series than for the $\mathrm{BF}_{4}$ series agree with the observation of Beichel et al., ${ }^{37}$ who also observed a systematic increase in the critical volumes for viscosity with the molecular size of the anion in the case of the $\left[\mathrm{C}_{4} \mathrm{MIM}\right]$ series with different anions. Based on the values from Ref. 37, the ratio of critical free volumes for the viscosity of Bmim TFSI and $\mathrm{Bmim} \mathrm{BF}_{4}$ is 1.23 .

The three transport properties of ILs studied, the translational diffusion of ${ }^{14} \mathrm{~N}$-pDTEMPONE cation and anion diffusion, and viscosity in TFSI and $\mathrm{BF}_{4}$ RTILs, can be described well in terms of free volume concepts. The fits of the experimental data to the CohenTurnbull equations [Eqs. (3) and (5)] are excellent. The goodness of fits validates the empirical procedure for finding the free volume in ILs proposed by Beichel et al.

Even in the cases when the self-diffusion coefficients of the cation and anion, e.g., Emim TFSI and Bmim TFSI [Fig. 4(b)], are noticeably different, the critical free volumes for the diffusion of the cation and anion are the same. The similarity of the critical free volumes for the cation and anion suggests that the diffusion of each ionic pair is coordinated. The ratio of critical free volumes between the two RTIL series for a given cation is similar for all three transport properties, and it appears close to the ratio of their densities.

\section{SUPPLEMENTARY MATERIAL}

See the supplementary material for EPR spectra and fits of ${ }^{14} \mathrm{~N}$-pDTEMPONE in $\mathrm{Emim}_{\mathrm{BF}}$, average spin dephasing rate $\Gamma$ and coherence transfer rate $\Lambda$ of ${ }^{14} \mathrm{~N}$-pDTEMPONE in Emim $\mathrm{BF}_{4}$, concentration coefficients $\mathrm{W}_{2}$ and $\mathrm{V}_{2}$ of ${ }^{14} \mathrm{~N}$-pDTEMPONE in Emim $\mathrm{BF}_{4}$, diffusion coefficient $D$ of ${ }^{14} \mathrm{~N}$-pDTEMPONE as a function of $T$, ratio of the density and free volume of TFSI and $\mathrm{BF}_{4}$ RTIL as a function of $T$, summation of the self-diffusion coefficients $D_{+}$and $D_{-}$as a function of $T$, molecular weights and scaled molecular volumes of RTILs, and densities of RTILs.

\section{ACKNOWLEDGMENTS}

This work was supported by the Croatian Science Foundation under Project Nos. 1108 and 3168, as well as by the Foundation of the Croatian Academy of Sciences and Arts. D.M. wishes to thank Dejana Carić for her assistance in sample preparation. M.P. gratefully acknowledges support from NSF MRI (Grant No. 1626632) and NSF RUI (Grant No. 1856746). 


\section{REFERENCES}

${ }^{1}$ J. G. Huddleston, H. D. Willauer, R. P. Swatloski, A. E. Visser, and R. D. Rogers, Chem. Commun. 1998, 1765.

${ }^{2}$ S. N. Baker, G. A. Baker, and F. V. Bright, Green Chem. 4, 165 (2002).

${ }^{3}$ N. Ito and R. Richert, J. Phys. Chem. B 111, 5016 (2007).

${ }^{4}$ D. Bankmann and R. Giernoth, Prog. Nucl. Mag. Reson. Spectr. 51, 63 (2007).

${ }^{5}$ M. J. Earle and K. R. Seddon, Pure Appl. Chem. 72, 1391 (2000).

${ }^{6}$ N. V. Plechkova and K. R. Seddon, Chem. Soc. Rev. 37, 123 (2008).

${ }^{7}$ M. Freemantle, Chem. Eng. News 82, 44 (2004).

${ }^{8}$ C. M. Gordon, Appl. Catal., A 222, 101 (2001).

${ }^{9}$ P. Wasserscheid and W. Keim, Angew.Chem., Int. Ed. 39, 3772 (2000).

${ }^{10}$ M. Armand, F. Endres, D. R. MacFarlane, H. Ohno, and B. Scrosati, Nat. Mater. 8, 621 (2009).

${ }^{11}$ S. Carda-Broch, A. Berthod, and D. W. Armstrong, Anal. Bioanal. Chem. 375, 191 (2003).

${ }^{12}$ B. Kirchner, Topics in Current Chemistry (Springer, Heidelberg, 2009), Vol. 290.

${ }^{13}$ A. Noda, K. Hayamizu, and M. Watanabe, J. Phys. Chem. B 105, 4603 (2001).

${ }^{14}$ H. Tokuda, S. Tsuzuki, M. A. B. H. Susan, K. Hayamizu, and M. Watanabe, J. Phys. Chem. B 110, 19593 (2006).

${ }^{15}$ H. Tokuda, K. Hayamizu, K. Ishii, M. A. B. H. Susan, and M. Watanabe, J. Phys. Chem. B 108, 16593 (2004).

${ }^{16}$ K. R. Harris and M. Kanakubo, Phys. Chem. Chem. Phys. 17, 23977 (2015).

${ }^{17}$ K. R. Harris and M. Kanakubo, J. Phys. Chem. B 120, 12937 (2016).

${ }^{18}$ A. Kaintz, G. Baker, A. Benesi, and M. Maroncelli, J. Phys. Chem. B 117, 11697 (2013).

${ }^{19}$ J. C. Araque, S. K. Yadav, M. Shadeck, M. Maroncelli, and C. J. Margulis, J. Phys. Chem. B 119, 7015 (2015).

${ }^{20}$ G. E. Pake and T. R. Tuttle, Phys. Rev. Lett. 3, 423 (1959).

${ }^{21}$ Y. N. Molin, K. M. Salikhov, and K. I. Zamaraev, Spin Exchange Principles and Applications in Chemistry and Biology (Springer, Berlin, 1980).
${ }^{22}$ B. Berner and D. Kivelson, J. Phys. Chem. 83, 1406 (1979).

${ }^{23}$ B. L. Bales and M. Peric, J. Phys. Chem. B 101, 8707 (1997).

${ }^{24}$ D. Merunka, M. Peric, and M. Peric, J. Phys. Chem. B 119, 3185 (2015).

${ }^{25}$ B. L. Bales and M. Peric, J. Phys. Chem. A 106, 4846 (2002).

${ }^{26}$ I. Peric, D. Merunka, B. L. Bales, and M. Peric, J. Phys. Chem. B 118, 7128 (2014).

${ }^{27}$ M. Peric, B. L. Bales, and M. Peric, J. Phys. Chem. A 116, 2855 (2012).

${ }^{28}$ D. Merunka and M. Peric, J. Phys. Chem. B 121, 5259 (2017).

${ }^{29}$ K. M. Salikhov, Appl. Magn. Reson. 38, 237 (2010).

${ }^{30}$ K. M. Salikhov, Appl. Magn. Reson. 47, 1207 (2016).

${ }^{31}$ D. Merunka and M. Peric, J. Mol. Liq. 277, 886 (2019).

${ }^{32}$ J. M. Zielinski and J. L. Duda, AIChE J. 38, 405 (1992).

${ }^{33}$ F. Faupel, W. Frank, M.-P. Macht, H. Mehrer, V. Naundorf, K. Rätzke, H. R. Schober, S. K. Sharma, and H. Teichler, Rev. Mod. Phys. 75, 237 (2003).

${ }^{34}$ A. K. Doolittle, J. Appl. Phys. 22, 1471 (1951).

${ }^{35}$ M. H. Cohen and D. Turnbull, J. Chem. Phys. 31, 1164 (1959).

${ }^{36} \mathrm{D}$. Turnbull and M. H. Cohen, J. Chem. Phys. 34, 120 (1961).

${ }^{37}$ W. Beichel, Y. Yu, G. Dlubek, R. Krause-Rehberg, J. Pionteck, D. Pfefferkorn, S. Bulut, D. Bejan, C. Friedrich, and I. Krossing, Phys. Chem. Chem. Phys. 15, 8821 (2013).

${ }^{38}$ G. Dlubek, Y. Yu, R. Krause-Rehberg, W. Beichel, S. Bulut, N. Pogodina, I. Krossing, and C. Friedrich, J. Chem. Phys. 133, 124502 (2010).

${ }^{39}$ Y. Yu, W. Beichel, G. Dlubek, R. Krause-Rehberg, M. Paluch, J. Pionteck, D. Pfefferkorn, S. Bulut, C. Friedrich, N. Pogodina, and I. Krossing, Phys. Chem. Chem. Phys. 14, 6856 (2012).

${ }^{40} \mathrm{Y}$. Yu, D. Bejan, and R. Krause-Rehberg, Fluid Phase Equilib. 363, 48 (2014).

${ }^{41}$ H. Petrache, S. E. Feller, and J. F. Nagle, Biophys. J. 70, 2237 (1997).

${ }^{42}$ Y. Wang and G. A. Voth, J. Am. Chem. Soc. 127, 12192 (2005).

${ }^{43}$ C. Lopes, N. A. José, and A. A. H. Pádua, J. Phys. Chem. B 110, 3330 (2006).

${ }^{44}$ R. Hayes, G. G. Warr, and R. Atkin, Chem. Rev. 115, 6357 (2015). 